CLINICAL STUDY

\title{
Evaluation of insulin resistance in a cohort of HIV-infected youth
}

\author{
Raffaella Rosso, Arianna Parodi ${ }^{1}$, Giuseppe d'Annunzio ${ }^{1}$, Francesca Ginocchio, Laura Nicolini, Chiara Torrisi ${ }^{1}$, \\ Maria Pia Sormani ${ }^{2}$, Renata Lorini ${ }^{1}$, Claudio Viscoli and Marina Vignolo ${ }^{1}$ \\ Department of Infectious Diseases, San Martino Hospital, University of Genoa, Largo R. Benzi 10, 16132 Genoa, Italy, ${ }^{1}$ Paediatric Clinic, G. Gaslini \\ Institute, University of Genoa, Largo G. Gaslini 5, 16147 Genoa, Italy and ${ }^{2}$ Biostatistics Unit, Department of Health Sciences, San Martino Hospital, \\ University of Genoa, Via Pastore, 16132 Genoa, Italy \\ (Correspondence should be addressed to R Rosso; Email: raffaella.rosso@unige.it)
}

\begin{abstract}
Objective: Metabolic abnormalities, including impairment of glucose homeostasis, have been well characterized in HIV-infected patients. In contrast to adults, insulin resistance and diabetes mellitus appear to be relatively uncommon finding in youth.

Design: We assessed insulin resistance, and associated risk factors, in a population of vertically HIV-infected children and young adults, when compared with a control population of healthy children. Methods: At the time of enrolment, weeks of pregnancy, birth weight, sex, age, weight, height, body mass index (BMI), pubertal stages, CDC classification, blood pressure, clinical lipodystrophy, hepatitis B or C co-infection, antiretroviral therapy, CD4 T lymphocyte counts, and HIV-RNA levels were recorded. Fasting plasma glucose and insulin levels and homeostatic model assessment-insulin resistance (HOMA-IR) were determined. These parameters were compared between HIV patients and healthy controls with multivariate analyses.

Results: Fasting insulin levels $(\mathrm{OR}=1.21, P<0.001)$ and glycemia $(\mathrm{OR}=0.89, P<0.001)$ were significantly different between HIV-infected patients and controls. Antiretroviral therapy duration $(r=0.281, P<0.05)$, triglyceride levels $(r=0.286, P<0.05)$, age $(r=0.299, P<0.05)$, and BMI SDS $(r=0.485, P<0.001)$ were significant predictor variables of insulin resistance, expressed as HOMAIR. Moreover, clinical lipodystrophy seems to be strongly correlated to glycemia $(P<0.05)$, triglyceride levels $(P<0.05)$, serum insulin levels $(P<0.001)$, HOMA-IR $(P<0.05)$, and also with therapy duration $(P<0.05)$.

Conclusions: Both HIV infection and antiretroviral therapy demonstrate differential effects on glucose metabolism in HIV-infected children. Targeted prevention of insulin resistance and diabetes mellitus in HIV-infected children and young adults is needed in order to avoid the associated long-term complications that would otherwise occur, given the improvement in life expectancy of HIV-infected individuals.
\end{abstract}

European Journal of Endocrinology 157 655-659

\section{Introduction}

Disorders of glucose metabolism ranging from reduction in insulin sensitivity to impaired glucose tolerance (IGT) and diabetes mellitus has recently been recognized in HIV-infected patients (1-4). The condition appears to be multifactorial in cause, involving adverse effects of antiretroviral medications and HIV-related viral and immunologic factors, as well as genetic influences, physical inactivity, and diet $(5,6)$.

Metabolic abnormalities, including IGT, dyslipidemia, and alterations in body fat distribution, have not been characterized in children to the same extent as in adults (1). In contrast to adults, insulin resistance (IR) and diabetes mellitus appear to be relatively uncommon in children (2-4). The standard technique for assessment of insulin sensitivity is the hyperinsulinemic-euglycemic clamp, which is often combined with the hyperglycemic clamp to determine the adequacy of compensatory $\beta$-cell hypersensitivity $(7,8)$. Several fasting or 'homeostatic' models have been proposed as non-invasive measurement techniques for insulin sensitivity, with a relatively good correlation with clamp techniques (9-11). The homeostatic model assessment-IR (HOMA-IR), fasting glucose/insulin ratio (FGIR), and quantitative insulin sensitivity check index (QUICKI) methods have been most frequently used in clinical investigations (12). As a measure of IR among childhood, HOMA-IR has been used more often than QUICKI and FGIR, although the pediatric cutoff is unclear (12-15). The aim of the present study was to assess IR, and risk factors associated, in a population of vertically HIV-infected 
children and young adults, when compared with a control population of healthy children.

\section{Subjects and methods}

\section{Patient sample}

All vertically HIV-infected children followed by the Infectious Diseases Clinic, San Martino Hospital, University of Genoa, Italy were included in the study. No patient had a family history of diabetes or IGT. At the time of enrolment, the following parameters were recorded: weeks of pregnancy, body weight at birth $(\mathrm{kg})$, sex, age (years), weight $(\mathrm{kg})$, height $(\mathrm{cm})$, body mass index (BMI, calculated as weight $(\mathrm{kg})$ divided by the height $\left(\mathrm{m}^{2}\right)$ ), BMI standard delta score units (SDS), and pubertal stage according to Tanner classification and blood pressure $(\mathrm{mmHg})$. BMI was calculated and expressed as a continuous variable in SDS, determined as the difference between the individual observed value and the reference mean for age and sex, divided by the corresponding S.D. In this way, the modifications of BMI according to age and sex can be taken into account.

Presence of hepatitis B or C co-infection (based on serological examination and RNA or DNA viral blood levels) as added risk factors for IR, CDC classification, and detailed histories of antiretroviral therapy were also recorded $(16,17)$. Fasting plasma glucose $(\mathrm{FPG}, \mathrm{mg} / \mathrm{dl})$ and fasting plasma insulin (FPI, $\mu \mathrm{U} / \mathrm{ml}$ ) levels were measured from serum samples. FPI was measured using an immunoradiometric method. The FGIR ratio was calculated and HOMA-IR was determined as (FPI $(\mu \mathrm{U} / \mathrm{ml}) \times \mathrm{FPG}(\mathrm{mmol} / \mathrm{l})) / 22.5)$. FGP, fasting triglycerides (TG, normal values $40-170 \mathrm{mg} / \mathrm{dl}$ ), fasting total cholesterol (CHO, normal values 130-200 mg/dl), LDL and HDL CHO $(\mathrm{mg} / \mathrm{dl})$, and lactate levels $(\mathrm{mmol} / \mathrm{l})$ were measured by standard laboratory assays. Clinical evaluations, by the same clinician, also included the assessment of lipodystrophy. It was classified as follows: a) clinical lipoatrophy if there was fat loss in the face (sunken cheeks), arms, legs, or buttocks; b) clinical lipohypertrophy if there was enlargement of the breasts, increased abdominal girth or dorsocervical fat accumulation; and c) mixed pattern if both peripheral atrophy and central accumulation of fat occurred (18).

\section{Controls}

For comparison, 138 unrelated normal-weight and normal-height children ( 84 males and 54 females, aged 3-19 years, median $11 \pm 3.8$ ), belonged to the same geographic area, were recruited as controls. They consulted the Gaslini Institute for presumed diseases (fever) and were found to be normal. All parents gave written informed consent; the study was approved by the local ethics committee.

\section{Statistical analyses}

HIV-infected case and healthy control characteristics were compared using a univariate and a multivariate logistic regression analysis with HIV status as the dependent variable; the multivariate model included age, sex, pubertal state, BMI SDS score, FPG, and FPI. The relationships between IR and clinical characteristics of HIV-infected cases were evaluated by correlating HOMA-IR index with baseline characteristics of HIV patients, using the Pearson correlation coefficient, at univariate analysis and a multiple linear regression for the multivariate analysis.

\section{Results}

The demographic and clinical data at baseline for HIVinfected patients and controls are shown in Table 1. All patients and controls were Caucasian. The majority of HIV patients were antiretroviral experienced and, according to the definitions given previously, lipodystrophy was observed in 33\% (16 out of 48) of the children: lipoatrophy $8 \%$, lipohypertrophy $6 \%$, and mixed pattern $19 \% ; 14.6 \%$ (7 out of 48 ) and $16.7 \%$ ( 8 out of 48 ) of children had elevated values of TG (median $89.5 \mathrm{mg} / \mathrm{dl}$, range 30-662) and CHO (median $230 \mathrm{mg} / \mathrm{dl}$, range 210-249) respectively. Median values of HDL and LDL CHO were $47.5 \mathrm{mg} / \mathrm{dl}$ (range 11-89) and $100.5 \mathrm{mg} / \mathrm{dl}$ (range 32-151) respectively. For HIV-infected children, mean weeks of pregnancy were 38.9 (median 40; range 31-42; S.D. 2.55) and the mean body weight at birth was $3.05 \mathrm{~kg}$ (median $3.15 \mathrm{~kg}$; range 1.16-5; S.D. 0.74). No mother received any kind of prophylaxis during pregnancy. FPG was normal in all HIVinfected subjects, ranging from 50 to $91 \mathrm{mg} / \mathrm{dl}$, while hyperinsulinemia (normal values $2-25 \mu \mathrm{U} / \mathrm{ml}$ ) was found in 29 out of $48(60.4 \%)$ HIV-infected subjects. FGIR lower than 7 , usually considered as a cutoff value for IR (12), was present in 26 out of 48 (52\%) HIV-infected children and 19 out of $138(16 \%)$ healthy children respectively. As shown in Table 2, there were no significant differences in sex, pubertal stage, and BMI SDS, between HIV patients and controls, while age was significantly higher in cases $(\mathrm{OR}=1.11, P=0.01)$ than in controls. FPG was significantly lower in HIV-infected patients than in control $(\mathrm{OR}=0.88, P<0.001)$, while FPI was significantly higher in HIV-infected patients than in controls $(\mathrm{OR}=1.19$, $P<0.001)$. After multiple regression analysis, adjusting for age, pubertal status, sex, and BMI SDS score, FPG $(\mathrm{OR}=0.89, \mathrm{P}<0.001)$ and FPI $(\mathrm{OR}=1.21, P<0.001)$ remained significantly different in HIV case and healthy controls. In HIV-infected children, therapy duration $(r=0.281, P=0.05)$, TG $(r=0.286, P=0.049)$, age $(r=0.299, \quad P=0.039)$, and BMI SDS $(r=0.485$, $P<0.001)$ were significantly correlated with IR; HOMAIR was also significantly different between those patients presenting (median 3.3, range 1.8-5.8) and not presenting (median 1.9, range 0.3-5.8) clinical lipodystrophy 
Table 1 Baseline characteristics of HIV-infected patients and healthy controls ${ }^{\mathrm{a}}$.

\begin{tabular}{|c|c|c|}
\hline Baseline characteristics & Patients & Controls \\
\hline No. of patients & 48 & 138 \\
\hline Median age in years (range) & $12(2-22)$ & $11(2.7-19)$ \\
\hline Sex (male/female) & $27 / 21$ & $54 / 84$ \\
\hline Average BMI SDS (S.D.) & $0.01(1.2)$ & $0.22(1.0)$ \\
\hline Tanner (pubescent/postpubertal) & $18 / 30$ & $68 / 70$ \\
\hline Median FPI in $\mu \mathrm{U} / \mathrm{ml}$ (range) & $11.5(2.0-32.0)$ & $6.15(1.7-20.1)$ \\
\hline Median FPG in mg/dl (range) & $78.5(50-91)$ & $85(64-100)$ \\
\hline Median FPI/FPG in $(\mathrm{mg} / \mathrm{dl}) /(\mu \mathrm{U} / \mathrm{ml})$ (range) & $6.4(2.2-25.0)$ & $13.4(3.9-51.8)$ \\
\hline Median HOMA-IR in $(\mu \mathrm{U} / \mathrm{ml}) \times(\mathrm{mmol} / \mathrm{l})$ (range) & $2.18(0.25-5.79)$ & $1.3(0.2-5.4)$ \\
\hline \multicolumn{3}{|l|}{ CDC classification } \\
\hline $\mathrm{N}$ & $2(4.2 \%)$ & \\
\hline A & $16(33.3 \%)$ & \\
\hline B & $23(47.9 \%)$ & \\
\hline C & $7(14.6 \%)$ & \\
\hline No. of co-infected patients (HCV versus HBV) & $10(20.8 \%)(9$ vs 1$)$ & \\
\hline No. of patients on antiretroviral therapy & $45(93.8 \%)$ & \\
\hline \multicolumn{3}{|l|}{ Type of therapy } \\
\hline 2 NRTIs & $9(18.8 \%)$ & \\
\hline HAART & $36(75.0 \%)$ & \\
\hline Pls-based & $20(55.5 \%)$ & \\
\hline NNRTI-based & $14(38.9 \%)$ & \\
\hline$N(t) R T I s-b a s e d$ & $2(5.6 \%)$ & \\
\hline Median cumulative duration of any therapy in months $(\text { range })^{b}$ & $105(1-188)$ & \\
\hline Median cumulative duration of HAART in months (range) ${ }^{b}$ & $61(3-85)$ & \\
\hline Median time of exposure to stavudine in months (range) ${ }^{b, c}$ & $57(9-76)$ & \\
\hline Median time of exposure to didanosine in months (range) $)^{\mathrm{b}, \mathrm{c}}$ & $50(2-81)$ & \\
\hline
\end{tabular}

$\mathrm{N}(\mathrm{t}) \mathrm{RTI}$, nucleoside (nucleotide) reverse transcriptase inhibitors; HAART, highly active antiretroviral therapy; PIs, protease inhibitors; NNRTI, non-nucleoside reverse transcriptase inhibitors.

${ }^{a}$ Number are expressed as median values for the groups.

${ }^{\mathrm{b}}$ Duration are expressed in months.

Including patients who had stopped it for $<6$ months before the start of the study.

$(P=0.009)$. Besides, comparing patients with and without lipodystrophy, the first had higher TG (median 122, range 72-662 versus median 69, range 30-659), FPI (median 19.6, range 8.5-32.4 versus median 9.85, range 3.4-30.4), and longer therapy duration (median 142, range $86-188$ versus median 81 , range $0-182$ ). Including these variables in a multivariate regression with a stepwise selection procedure, only presence of lipodystrophy $(P=0.009)$ and BMI SDS $(P=0.045)$ remained significantly associated with HOMA-IR. No correlations were found between the type of antiretroviral therapy (protease inhibitors (PIs)-based versus non-nucleoside reverse transcriptase inhibitors (NNRTI)-based versus nucleoside (nucleotide) reverse transcriptase inhibitors
$(\mathrm{N}(\mathrm{t}) \mathrm{RTI})$-based) and FPG, FPI, or HOMA-IR. Both HCV or HBV infection and CDC classification were not correlated with HOMA-IR.

\section{Discussion}

Although IR has been widely reported to occur among HIV-infected patients, this condition has not been well characterized in HIV-infected children and adolescents. The results of previous pediatric studies suggest that IR is relatively uncommon in HIV-infected children receiving antiretroviral therapy $(3,4,19)$. In these studies, IR diagnosis is based on elevated fasting

Table 2 Logistic regression analysis comparing HIV patients and controls.

\begin{tabular}{|c|c|c|c|c|c|c|c|}
\hline & \multirow[b]{2}{*}{$\begin{array}{l}\text { Unit of } \\
\text { measure }\end{array}$} & \multicolumn{3}{|c|}{ Univariate analysis } & \multicolumn{3}{|c|}{ Multivariate analysis } \\
\hline & & OR & $(95 \% \mathrm{Cl})$ & $P$ & OR & $(95 \% \mathrm{Cl})$ & $P$ \\
\hline \multirow[t]{2}{*}{ Sex } & $M$ & 1.00 & (ref) & & 1 & (ref) & \\
\hline & $\mathrm{F}$ & 1.21 & $(0.62-2.35)$ & 0.57 & 0.90 & $(0.38-2.11)$ & 0.81 \\
\hline \multirow[t]{2}{*}{ Tanner } & Pubescent & 1.00 & (ref) & & 1 & (ref) & \\
\hline & Postpubertal & 1.62 & $(0.83-3.17)$ & 0.16 & 0.40 & $(0.11-1.45)$ & 0.17 \\
\hline Age & Years & 1.11 & $(1.02-1.21)$ & 0.01 & 1.06 & $(0.92-1.23)$ & 0.41 \\
\hline BMI SDS & Z-score & 0.82 & $(0.59-1.13)$ & 0.23 & 0.78 & $(0.51-1.19)$ & 0.25 \\
\hline FPI & $\mu \mathrm{U} / \mathrm{ml}$ & 1.19 & $(1.11-1.27)$ & $<0.001$ & 1.21 & $(1.11-1.32)$ & $<0.001$ \\
\hline FPG & $\mathrm{mg} / \mathrm{dl}$ & 0.88 & $(0.84-0.93)$ & $<0.001$ & 0.89 & $(0.83-0.94)$ & $<0.001$ \\
\hline
\end{tabular}


insulinemia levels, while the use of clamp technology and/or fasting HOMA-IR is limited. Our results, based on the fasting HOMA-IR to evaluated IR, show that although HOMA-IR was higher in the HIV group, fasting glucose levels were normal in all; but it is relevant to highlight that patients with IR tend to remain euglycemic as long as there is an adequate compensatory increase in insulin output from the pancreas. IGT and diabetes ensue when the level of IR exceeds the compensatory increase in pancreatic insulin output.

Similar results, as regards HOMA-IR, are also reported in other small studies, but when evaluation was repeated after a 2-year interval, HOMA-IR values in HIV-infected group surprisingly decreased and were no longer different from healthy controls (20).

It has been suggested that several factors may contribute to the risk of developing IR in these subjects, including type and duration of antiretroviral agents, severity and duration of HIV infection, undetectable viral load, age, weight, BMI, HCV infection, and lipid abnormalities (21), but the small sample size of the pediatric studies have limited power to detect factors related to impairment of glucose homeostasis.

In our study, IR was not associated with type and duration of HAART, N(t)RTI (particularly didanosine and stavudine), and use or duration of PIs (particularly lopinavir/ritonavir and nelfinavir). Although many pediatric studies failed to demonstrate an association of FPI or HOMA-IR with PIs therapy $(3,22)$, it has been fairly well established that PIs are linked with pathogenesis of IR in HIV-infected adults and the same processes are occurring in children, but are less apparent clinically. As recently reported by Bitnun et al. (23) only stimulated insulin sensitivity (derived from the frequent sampling i.v. glucose-tolerance test) has allowed detection of early disturbances in glucose homeostasis in children treated with PIs. Furthermore, it should be taken into account that each PI differs in its ability to induce IR: the present study was based on a small number of HIV-infected children and young adults, and patients were not randomly assigned to receive different types of therapy or PIs. Thus, it is lacking in statistical power to demonstrate an association between PI use and IR.

We found a clear correlation between IR and duration of antiretroviral treatment: this result agrees with several studies and shows that time of exposure to antiretrovirals is an important associated risk factor to the development of HIV-related metabolic abnormalities $(24,25)$. IR may not only arise after longer therapy duration, but it may also be a simple illness and/or agerelated effect: all patients of the present study were vertically HIV-infected and started on antiretroviral therapy at early ages.

The recent follow-up study by Beregszaszi et al. (24). showing a doubling of the number of HIV-infected children with IR after 2 years of prospective observation, seems to confirm that reduced insulin sensitivity may develop progressively with increasing age and puberty. Pubertal development is considered a critical period for IR, since during puberty a transition to a less insulin-sensitive state occurs mediated, at least partly, by hormonal changes $(26,27)$.

As expected, our patients, presented with clinical lipodystrophy, had, higher FPI and HOMA-IR and also therapy duration. This may suggest that increased visceral fat storage with a deposit pattern leading to lipodystrophy with central fat accumulation in the abdomen/posterior neck is a predicting factor for IR. Sanchez Torres et al. (28) have recognized different clinical patterns of fat redistribution in HIV-infected children with lipohypertrophy, characterized mainly by abdominal fat accumulation, being the commonest pattern found in children and probably the first phase of a progressive process of fat redistribution leading to lipoatrophy.

As a part of this process, dyslipidemia with hypertriglyceridemia, with or without hypercholesterolemia (and elevated LDL fractions), and peripheral IR are frequently reported. We found significant associations between FPI, HOMA-IR, and TG, but no significant association with $\mathrm{CHO}$ and LDL fraction. It has to be pointed out that a large proportion of subjects in our series showed hypercholesterolemia without hypertriglyceridemia and IR; moreover, metabolically TG are more closely related to glucose homeostasis than cholesterol.

In contrast with previous data showing a higher risk of IR in HCV co-infected patients, in our patients both $\mathrm{HCV}$ and HBV co-infections were not correlated with IR (29).

Finally, some limitations in this cross-sectional study have to be recognized: first the sample size and therapeutic heterogeneity of the patients are limited; secondly, the majority of patients in the study were postpubertal, and this would make them metabolically more like adults than prepubertal children. The differences in results of this paper and those of other pediatric studies may, at least in part, be related to this; and thirdly, lipodystrophy was evaluated subjectively by general clinical appearance rather than objectively using dual-energy X-ray absorptiometry and/or magnetic resonance imaging, or skinfold thickness or biometrical impedance measurements.

In conclusion, although the natural history of HIVrelated metabolic changes are still poorly known, they may be considered as progressive processes, related to HIV infection, antiretroviral therapy, and probably individual or genetic predisposing factors. In this context, early recognition and management of IR become an important aspect of long-term health maintenance for HIV-infected children and young adults. In addition, we would focus on the use of FPI and HOMA-IR as measures of utility in routine clinical care, while we think that clamp technology and i.v. glucose tolerance testing, because of their complexity, are less clinically relevant but more useful in research. 


\section{References}

1 Florescu D \& Kotler DP. Insulin resistance, glucose intolerance and diabetes mellitus in HIV infected patients. Antiviral Therapy 2007 12 149-162.

2 Taiwo BO. Insulin resistance, HIV infection and anti-HIV therapies. AIDS Reader 200515 171-176 (179-180).

3 Bitnun A, Sochett E, Babyn P, Holowka S, Stephens D, Read S \& King SM. Serum lipids, glucose homeostasis and abdominal adipose tissue distribution in protease inhibitor-treated and naïve HIV-infected children. AIDS 200317 1319-1327.

4 Lainka E, Oezbek S, Falck M, Ndagijjmana J \& Niehues T. Marked dyslipidemia in human immunodeficiency virus-infected children on protease inhibitor-containing antiretroviral therapy. Pediatrics 2002110 e56.

5 Leow MK, Addy CL \& Mantzoros CS. Clinical review 159: human immunodeficiency virus/highly active antiretroviral therapyassociated metabolic syndrome: clinical presentation, pathophysiology, and therapeutic strategies. Journal of Clinical Endocrinology and Metabolism $2003 \mathbf{8 8}$ 1961-1976.

6 Tomazic J, Karner P, Vidmar L, Maticic M, Sharma PM \& Janez A. Effect of metformin and rosiglitazone on lipid metabolism in HIV infected patients receiving protease inhibitor containing HAART. Acta Dermatovenerologica Alpina, Panonica, et Adriatica 200514 99-105.

7 Gungor N, Saad R, Janosky J \& Arslanian S. Validation of surrogate estimates of insulin sensitivity and insulin secretion in children and adolescents. Journal of Pediatrics 2004144 47-55.

8 Haymond MW. Measuring insulin resistance: a task worth doing. But how? Pediatric Diabetes 20034 115-118.

9 Silfen ME, Manibo AM, McMahon DJ, Levine LS, Murphy AR \& Oberfield SE. Comparison of simple measures of insulin sensitivity in young girls with premature adrenarche: the fasting glucose to insulin ratio may be a simple and useful measure. Journal of Clinical Endocrinology and Metabolism 200186 2863-2868.

10 Laakso M. How good a marker is insulin level for insulin resistance? American Journal of Epidemiology 1993137 959-965.

11 Quon MJ. Limitations of the fasting glucose to insulin ratio as an index of insulin sensitivity. Journal of Clinical Endocrinology and Metabolism 200186 4615-4617.

12 Keskin M, Kurtoglu S, Kendirci M, Atabek ME \& Yazici C. Homeostasis model assessment is more reliable than the fasting glucose/insulin ratio and quantitative insulin sensitivity check index for assessing insulin resistance among obese children and adolescents. Pediatrics 2005115 e500-e503.

13 Tresaco B, Bueno G, Pineda I, Moreno LA, Garagorri JM \& Bueno M. Homeostatic model assessment (HOMA) index cut-off values to identify the metabolic syndrome in children. Journal of Physiology and Biochemistry 200561 381-388.

14 Valerio G, Licenziati MR, Iannuzzi A, Franzese A, Siani P, Riccardi G \& Rubba P. Insulin resistance and impaired glucose tolerance in obese children and adolescents from Southern Italy. Nutrition, Metabolism, and Cardiovascular Diseases 200616 279-284.

15 Radikova Z, Koska J, Huckova M, Ksinantova L, Imrich R, Vigas M, Trnovec T, Langer P, Sebokova E \& Klimes I. Insulin sensitivity indices: a proposal of cut-off points for simple identification of insulin-resistant subjects. Experimental and Clinical Endocrinology and Diabetes $2006114249-256$.

16 Duong M, Petit JM, Piroth L, Grappin M, Buisson M, Chavanet P, Hillon P \& Portier $\mathrm{H}$. Association between insulin resistance and hepatitis $\mathrm{C}$ virus chronic infection in HIV-hepatitis $\mathrm{C}$ virus co-infected patients undergoing antiretroviral therapy. Journal of Acquired Immune Deficiency Syndromes 200127 245-250.
17 Laguno M, Milinkovic A, de Lazzari E, Murillas J, Martínez E, Blanco JL, Loncá M, Biglia A, Leon A, García M, Larrousse M, García F, Miró JM, Gatell JM \& Mallolas J. Incidence and risk factors for mitochondrial toxicity in treated HIV/HCV-coinfected patients. Antiviral Therapy 200510 423-429.

18 Guillén S, Ramos JT, Resino R, Bellón JM \& Muñoz MA. Impact on weight and height with the use of HAART in HIV-infected children. Pediatric Infectious Disease Journal 200726 334-338.

19 Amaya RA, Kozinetz CA, McMeans A, Schwarzwald H \& Kline MW. Lipodystrophy syndrome in human immunodeficiency virus-infected children. Pediatric Infectious Disease Journal 200221 405-410.

20 Rondanelli M, Caselli D, Trotti R, Solerte SB, Maghnie M, Maccabruni A, Minoli L \& Ferrari E. Endocrine pancreatic dysfunction in HIV-infected children: association with growth alterations. Journal of Infectious Diseases 2004190 908-912.

21 Wanke CA, Falutz JM, Shevitz A, Phair JP \& Kotler DP. Clinical evaluation and management of metabolic and morphologic abnormalities associated with human immunodeficiency virus. Clinical Infectious Diseases 200234 248-259.

22 Melvin AJ, Lennon S, Mohan KM \& Purnell JQ. Metabolic abnormalities in HIV type 1-infected children treated and not treated with protease inhibitors. AIDS Research and Human Retroviruses 200117 1117-1123.

23 Bitnun A, Sochett E, Dick PT, To T, Jefferies C, Babyn P, Forbes J. Read S \& King SM. Insulin sensitivity and beta-cells function in protease inhibitors-treated and -naïve human immunodeficency virus-infected children. Journal of Clinical Endocrinology and Metabolism 200590 168-174.

24 Beregszaszi M, Dollfus C, Levine M, Faye A, Deghmoun S, Bellal N, Houang M, Chevenne D, Hankard R, Bresson JL, Blanche S \& LevyMarchal C. Longitudinal evaluation and risk factors of lipodystrophy and associated metabolic changes in HIV-infected children. Journal of Acquired Immune Deficiency Syndromes $2005 \mathbf{4 0}$ $161-168$.

25 Leonard EG \& McComsey GA. Antiretroviral therapy in HIVinfected children: the metabolic cost of improved survival. Infectious Disease Clinics of North America 200519 713-729.

26 De Ridder CM, Thijssen JH, Bruning PF, Van den Brande JL, Zonderland ML \& Erich WB. Body fat mass,body fat distribution, and pubertal development: a longitudinal study of physical and hormonal sexual maturation of girls. Journal of Clinical Endocrinology and Metabolism 199275 442-446.

27 Moran A, Jacobs DR, Steinberger J, Hong CP, Prineas R, Luepker R \& Sinaiko AR. Insulin resistance during puberty: results from clamp studies in 357 children. Diabetes 199948 2039-2044.

28 Sanchez Torres AM, Munoz Muniz R, Madero R, Borque C, GarciaMiguel MJ \& De Jose Gomez MI. Prevalence of fat redistribution and metabolic disorders in human immunodeficiency virusinfected children. European Journal of Pediatrics $2005 \mathbf{1 6 4}$ 271-276.

29 de Larranaga GF, Wingeyer SD, Puga LM, Alonso BS \& Benetucci JA. Relationship between hepatitis $\mathrm{C}$ virus (HCV) and insulin resistance, endothelial perturbation, and platelet activation in HIV-HCV-coinfected patients under highly active antiretroviral treatment. European Journal of Clinical Microbiology and Infectious Diseases 200614 1-6.

Received 30 August 2007

Accepted 3 September 2007 